\title{
A Combination between Laplace Transform, Strip Method and Transition Matrix for Determination of Dynamic Response and Damping Effect of Plates
}

\author{
A.S. Ashour ${ }^{\dagger}$ and \\ Faculty of Engineering, International Islamic University Malaysia, 53100 Kuala Lumpur, Malaysia
}

\begin{abstract}
A.M. Farag
Department of Engineering Mathematics and Physics, Faculty of Engineering, Alexandria University, Alexandria, Egypt
\end{abstract}

(Received 5 September 1999; accepted 24 May 2000)

\begin{abstract}
The main objective of this paper is to assess the dynamic response of a plate under the action of a force having a general variation with time. An efficient and powerful numerical method for the determination of the dynamic response and the damping analysis of a rectangular plate subjected to lateral excitation is proposed. The present technique is accomplished by a combination of the finite strip, transition matrix, and Laplace transform with respect to time. The plate system is idealised as an assembly of a number of strip elements whose transition matrices are derived in the Laplace transformation domain. In the transformed domain, the problem is solved numerically by a finite strip transition matrix method, whereas the dynamic response is finally expressed in the time domain by a numerical inversion of the transformed solution. This study takes into account the effect of the internal viscoelastic damping and the partially restrained boundary conditions. The merits of the present methodology are demonstrated with suitable numerical examples.
\end{abstract}

${ }^{\dagger}$ Member of the International Institute of Acoustics and Vibration (IIAV)

\section{INTRODUCTION}

The transient response of composite plates to a dynamic load has been the subject of numerous investigations. Different approaches have been used in the literature to determine the dynamic response of such plates in various engineering fields. ${ }^{1-3}$ Among the popular methods, the modal superposition $^{4-6}$ and step-by-step methods, (so called direct integration methods) $)^{1,2}$ are often used. However, both of these methods require a large computer memory, especially if the use of finite elements, finite differences or strips are used to investigate plate response..$^{7-9}$ Moreover, a round-off error occurs during the solution, which eventually leads to numerical damping $^{1}$ (i.e. amplitude decay or period elongation). In order to overcome the disadvantages of the above methods, a Laplace transform with respect to time in conjunction with the finite strip-transition matrix method ${ }^{4,10}$ is used in the present approach.

Compared to either the finite element or the finite difference method, the finite strip technique requires a much smaller total number of degrees of freedom for the whole system. ${ }^{7,9,14}$ The Finite Strip-Transition Matrix Method (FSTM) has proved to be a very efficient and powerful numerical method to determine the response of rectangular skew, or sectorial plates bounded by any type of classical or restrained boundary conditions. ${ }^{4,10}$ In addition, the use of the Laplace transform has the advantages that the dynamic problem is effectively reduced to a static one. Another advantage appears when the external viscoelastic damping as well as forces having a general variation with time are investigated by the present technique. Based on these, one expects that a combination of the finite strip transition matrix and the Laplace transform to offer an accurate solution for the problems of the dynamic excitation of rectangular plates.

The plate system is divided into a number of strips, which are assembled and solved by the use of a transition matrix in the transformed domain. The dynamic response is finally achieved by a numerical inversion of the transformed solution. By employing a direct numerical Laplace transformation, forces having an arbitrary variation with time can easily be handled. Durbin's algorithm ${ }^{11}$ is exploited in the present work. This algorithm is considered to be one of the most accurate and convenient for both inverse and direct Laplace transformation computations of structural dynamics. ${ }^{12,13}$

\section{THEORETICAL IDEALISATION}

\subsection{Governing Equations}

The usual thin plate assumptions which govern the bending behaviour of rectangular plates whose material exhibits orthotropic properties are assumed in this investigation. The equation of motion of a plate subjected to a dynamic load function $F(x, y, t)$, external viscous damping of constant $c$ and which rests on a homogenous elastic sub-grade of modulus $K_{0}$ can be expressed as:

$$
D_{x} \frac{\partial^{4} w}{\partial x^{4}}+2 H_{x y} \frac{\partial^{4} w}{\partial x^{2} \partial y^{2}}+D_{y} \frac{\partial^{4} w}{\partial y^{4}}+M \frac{\partial^{2} w}{\partial t^{2}}+c \frac{\partial w}{\partial t}+K_{0} w=
$$

$$
F(x, y, t)
$$

International Journal of Acoustics and Vibration, Vol. 5, No. 4, 2000

(pp 191-195) 